\title{
PERFIL DOS PACIENTES ATENDIDOS NA BEBÊ CLIINICA DA FACULDADE DE ODONTOLOGIA DA UNIVERSIDADE FEDERAL DO RIO GRANDE DO SUL ENTRE OS ANOS DE 2006 E 2011
}

\author{
PROFILE OF PATIENTS TREATED AT THE $B A B Y C L I N I C$ OF THE \\ DENTISTRY SCHOOL AT THE FEDERAL UNIVERSITY OF RIO \\ GRANDE DO SUL FROM 2006 TO 2011
}

\begin{abstract}
Juliana Silveira Emerim¹; Luana Vieira Roque da Silva²; Mircelei Saldanha Sampaio $^{3}$; Márcia Cançado Figueiredo ${ }^{4}$

${ }^{1}$ Acadêmica do curso de graduação em Odontologia da Universidade Federal do Rio Grande do Sul, Porto Alegre, RS, Brasil. E-mail: <juliana2303@hotmail.com>

${ }^{2}$ Acadêmica do curso de graduação em Odontologia da Universidade Federal do Rio Grande do Sul, Porto Alegre, RS, Brasil. E-mail: <luana.vrs@hotmail.com>

${ }^{3}$ Membro da Equipe Executora da Extensão Universitária: Bebê Clínica da Faculdade de Odontologia da Universidade Federal do Rio Grande do Sul, Porto Alegre, RS, Brasil. E-mail: <mircesampaio@gmail.com>

${ }^{4}$ Professora Associada IV e Coordenadora da Extensão Universitária: Bebê Clínica da Faculdade de Odontologia da Universidade Federal do Rio Grande do Sul/UFRGS, Porto Alegre, RS, Brasil. E-mail: <mcf1958@gmail.com>
\end{abstract}

Data de recebimento: 02/08/2012

Data da aprovação: 25/09/2012

\section{RESUMO}

Descrever o perfil dos pacientes atendidos na Bebê Clínica da FO/UFRGS, a partir da análise dos registros de prontuários abertos em consultas iniciais realizadas nos anos de 2006 a 2011 foi o objetivo deste artigo, resultado do Trabalho de Conclusão de Curso da Faculdade de Odontologia da Universidade Federal do Rio Grande do Sul. Foram observadas crianças, cujas faixas etárias variavam entre 1 a 3 anos de idade, e o principal motivo da consulta foi a prevenção. Utilizou-se de análise estatística descritiva dos dados de 569 prontuários com auxílio do software SPSS (versão 17.0), recorrendo-se ao Teste Qui-quadrado para a obtenção das porcentagens das variáveis de comparação $(\mathrm{p}<0,05)$. Os resultados revelaram que $65,2 \%$ das crianças possuíam dieta cariogênica e $88,6 \%$ tinham sua higiene bucal realizada periodicamente. Em relação à atividade de cárie, 54,7\% não possuíam atividade, estando essa frequência presente principalmente nas faixas etárias de 0 a 1 ano $(96,1 \%)$ e de 1 a 2 anos $(61,1 \%)$. Assim, pôde-se concluir que aproximadamente metade dos pacientes possuía uma dieta cariogênica e atividade de cárie, apesar de a grande maioria dos responsáveis ter afirmado realizar a higiene de seus bebês com frequência e utilizar a escova dental.

Palavras-chave: Saúde bucal. Prevenção primária. Epidemiologia.

\begin{abstract}
Aim: Describe the profile of patients treated at the FO/UFRGS Baby Clinic based on the analysis of initial consultations records from 2006 to 2011. This article is the result of a
\end{abstract}


final paper presented at the Dentistry Course from the Federal University of Rio Grande do Sul. Children form 1 to 3 years old were observed and the main reason for the initial consultation was prevention of caries. Materials and Methods: descriptive statistical analysis of data from 569 charts with SPSS software (version 17.0), using the Chi-square test to obtain the percentages of comparison variables $(p<0.05)$. Results: the results demonstrate that $65.2 \%$ of the children followed a cariogenic diet and that $88.6 \%$ had their oral hygiene performed on a regular basis. Concerning caries activity, $54.7 \%$ of the children did not have caries activity; this percentage was present especially in children from 0 to 1 year old $(96.1 \%)$ and in children from 1 to 2 years old $(61.1 \%)$. Thus, it is possible to conclude that approximately half of the patients followed a cariogenic diet and had caries activity, despite most of the adults responsible for the children affirmed they performed the oral hygiene of the babies and used toothbrushes.

Keywords: Oral health. Primary prevention. Epidemiology.

\section{Introdução}

A odontologia para bebês, como forma de atenção precoce, é uma grande conquista e representa a incorporação de um novo entendimento na abordagem da saúde bucal. Focada na promoção e prevenção da saúde bucal, objetiva oferecer condições ideais para o correto desenvolvimento do sistema estomatognático da criança, uma vez que as condições de saúde bucal de cada indivíduo são estabelecidas na infância (FRACASSO, et al., 2008).

Constata-se que um dos motivos de maior procura de atendimento odontológico para bebês é a Cárie Precoce da Infância (ECC) que, segundo Jin et al. e Walter, Ferelle; Issao, possui uma característica agressiva, podendo causar quadros de muita dor, dificuldades de mastigação, problemas de fala, distúrbios na saúde geral, além de comprometimentos na qualidade de vida do bebê e da família (SAMPAIO, 2005).Não deveria constar a data nos autores citados no interior do parágrafo?

Apesar do declínio substancial da prevalência de cárie das últimas décadas (KOPYCKAKEDZIERAWSKI, 2008), os dados epidemiológicos existentes ainda são preocupantes. No Brasil essa prevalência pode ser verificada através dos dados do Levantamento Nacional de Saúde Bucal de 2003 (SB Brasil 2003) em que registraram que $27 \%$ dos bebês de 18 a 36 meses e $60 \%$ das crianças de 5 anos de idade apresentavam dentes cariados. No levantamento realizado em 2010 (SB Brasil 2010) não foram analisados dados referentes a bebês, impossibilitando uma avaliação ao longo do tempo nessa faixa etária. Porém, cabe salientar que, nesse levantamento, do total de crianças com 5 anos de idade que possuíam atividade de cárie, apenas $20 \%$ receberam tratamento odontológico.

Atentos a toda essa problemática da doença cárie em bebês e diante da escassez de programas odontológicos para essa faixa etária, foi instituída no início da década de 90 a Bebê Clínica da Faculdade de Odontologia da Universidade Federal do Rio Grande do Sul (Bebê Clínica FO/UFRGS) e, em 1995, a ação de extensão Bebê Clínica da FO/UFRGS. Este programa visa o atendimento odontológico a pacientes de 0 a 36 meses de idade, priorizando a faixa etária de 0 a 12 meses, com o objetivo de garantir que esses bebês fiquem livres da doença cárie ao longo de suas vidas. Sua criação teve como norte a Bebê Clínica da Universidade Estadual de Londrina, pioneira no Brasil, fundada em 1985 (WALTER, FERELLE; ISSAO,1996).

A Bebê Clínica da FO/UFRGS tem passado por um grande processo de transformação desde sua implantação. A equipe de professores e alunos tem buscado incessantemente a qualidade na atenção educativa/preventiva, no sentido de promover saúde e ampliar o acesso da população aos serviços oferecidos, visando a resolubilidade dos problemas odontológicos que acometem bebês. Nesse contexto, ao longo dos anos, os atendimentos, além de serem a pacientes que vêm por livre demanda, foram estendidos também àqueles oriundos das Unidades Básicas de Saúde (UBS) de Porto Alegre, devido ao convênio 
entre FO/UFRGS e Secretaria Municipal de Saúde de Porto Alegre (CEO-UFRGS/SUS).

Como forma de contribuir com todo esse processo de transformação e auxiliar no planejamento de ações de saúde para uma melhor qualidade deste programa, estudos epidemiológicos tornam-se de grande valia. Esses estudos objetivam descrever as condições de saúde da população, investigam os fatores determinantes das situações de saúde e avaliam o impacto dessas ações de saúde, proporcionando, através de seus resultados, a possibilidade de contribuir para uma melhor resolubilidade no âmbito da saúde (ESTRELA, 2001).

Dessa maneira, o presente estudo teve como objetivo descrever o perfil dos pacientes atendidos na Bebê Clínica FO/UFRGS a partir da análise dos registros de todos os prontuários abertos em consultas iniciais realizadas entre os anos de 2006 e 2011.

\section{Materiais e métodos}

Este foi um estudo descritivo com base em registros de prontuários. Após a aprovação pela Comissão de Pesquisa de Odontologia da FO/UFRGS e de Ética em Pesquisa da UFRGS, com protocolo número 10.113, foi feita a análise quantitativa de todos os 569 prontuários arquivados na Bebê Clínica da FO/UFRGS relativos a pacientes que ingressaram no programa entre os anos de 2006 e 2011 (6 anos), através da livre demanda ou encaminhados pelas UBS para o Centro de Especialidades Odontológicas (CEO- UFRGS/SUS).

Os prontuários analisados foram preenchidos por alunos de graduação e revisados por um dos professores da equipe da Bebê Clínica da FO/UFRGS durante os atendimentos clínicos. Os registros foram analisados por duas pesquisadoras, graduandas na FO/UFRGS. Os dados obtidos a partir desses prontuários contemplaram variáveis referentes a: identificação do bebê (sexo e idade); características sociodemográficas (procedência da família, quem era o acompanhante do bebê e seu grau de instrução); informações relativas à consulta (forma de ingresso no programa e motivo da consulta); história médica (informações sobre gestação, aleitamento até os 6 meses e saúde geral); história odontológica (consultas odontológicas pregressas e histórico de trauma); informações relevantes relacionadas à saúde bucal (hábitos deletérios e alimentares); informações sobre a higiene bucal; atividade de cárie; número de consultas e de tratamentos realizados.

As informações coletadas foram registradas em um banco de dados do programa EXCEL e, após, foi realizada a análise estatística descritiva com auxílio do software SPSS versão 17.0, buscando dados de frequências simples e percentuais das variáveis coletadas. A obtenção das porcentagens das variáveis de comparação foi feita com a utilização do Teste Qui-quadrado, em que os valores de $\mathrm{p}$ foram considerados estatisticamente significantes quando $<0,05$.

\section{Resultados}

inicialmente, é importante esclarecer que, devido ao fato deste estudo ser baseado em registros de prontuários, em algumas variáveis os valores totais serão menores que o $\mathrm{N}$ (569) por consequência de dados que não haviam sido preenchidos corretamente nos prontuários. Da totalidade de prontuários clínicos analisados, quanto à identificação dos bebês, pôde-se observar que $53,3 \%$ eram do sexo masculino e $46,7 \%$, feminino. Quanto à faixa etária, verificou-se que $20,4 \%$ tinham entre 0 e 1 ano; $37,1 \%$, entre 1 e 2 anos; $33,7 \%$, entre 2 e 3 anos e $8,8 \%$ tinham mais de 3 anos.

Quanto às características sociodemográficas, a maioria das famílias (74\%) residia em Porto Alegre, e $87,2 \%$ dos bebês foram acompanhados por suas mães. Com relação ao grau de instrução dos acompanhantes, 30,3\% tinham escolaridade até o ensino fundamental, $47,8 \%$ até o ensino médio e $21,9 \%$ até o ensino superior.

Quanto a informações relativas à consulta odontológica, verificou-se que $51,5 \%$ dos bebês ingressaram no programa por livre demanda e 48,5\%, foram encaminhados pelas UBS. Em relação ao motivo da consulta, $50,2 \%$ dos acompanhantes responderam que vieram à procura de prevenção, 28,8\% porque o bebê tinha cárie, $11,2 \%$ por traumatismo dentário, $4,2 \%$ por alterações em mucosa e $5,6 \%$ por anomalias dentárias (Tabela 1).

Quanto à história médica, foram analisadas informações sobre o período gestacional do bebê e a maioria das mães $(84 \%)$ teve uma gestação normal e um parto normal (53\%), salientando que a porcentagem de mães que fizeram cesárea (42,7\%) também 
foi expressiva. Quando indagadas sobre terem ou não uma dieta cariogênica (mais de 6 ingestas de açúcar por dia), $47,7 \%$ das mães relataram que tiveram tal dieta e 59,35\% não. Em relação ao aleitamento até os 6 meses, constatou-se que $70 \%$ dos bebês foram amamentados exclusivamente no peito, $7,7 \%$ foram amamentados com formulações (leite de vaca modificado) e 22,2\%, com a combinação de aleitamento materno mais formulações. Das respostas relacionadas ao fato de a criança possuir alguma doença sistêmica, $36,5 \%$ dos acompanhantes responderam positivamente, e as doenças mais prevalentes foram doenças respiratórias $(57,1 \%)$, alterações sanguíneas $(15,3 \%)$ e doenças infectocontagiosas da infância $(11,7 \%)$. Setenta e três por cento das crianças não possuíam alergia e 70,3\% não tomavam nenhum medicamento periodicamente. A maioria das crianças $(97,4 \%)$ tomaram todas as vacinas e, quanto ao estado de saúde da criança, $92,9 \%$ foram classificados como bom (Tabela 2).

Nos dados relacionados à história odontológica dos pacientes, identificou-se em $64,1 \%$ dos prontuários que aquela era a primeira consulta odontológica da vida do bebê, e a maioria deles $(79,3 \%)$ nunca sofreu nenhum tipo de traumatismo dentário.

Em relação aos hábitos bucais das crianças, $48 \%$ não possuíam nenhum hábito deletério (chupeta, dedo e outros) e $41,5 \%$ faziam uso de chupeta. Quanto ao tipo de alimentação que o bebê recebia, os dados observados foram: da totalidade dos que recebiam aleitamento materno, 48,3\% eram amamentados exclusivamente desta forma, enquanto que $51,7 \%$ recebiam também aleitamento complementado com formulações; e considerando todos os que não eram amamentados no peito, $93,1 \%$ recebiam apenas formulações e 6,9\% não recebiam nenhum tipo de aleitamento. Quanto ao período de aleitamento, $32 \%$ eram amamentados apenas no período diurno e $68 \%$ também eram amamentados no período noturno. Observou-se que os alunos consideraram que $65,2 \%$ dos bebês possuíam uma dieta cariogênica, e $34,8 \%$ não-cariogênica.

Quanto à higiene bucal dos bebês, a maioria dos acompanhantes $(88,6 \%)$ afirmou que era realizada periodicamente. Quando perguntados sobre quem executava essa higiene, $88,6 \%$ dos acompanhantes responderam que eram os próprios pais. Sobre a forma como ela era realizada, $17,5 \%$ dos pacientes tinham sua higiene bucal feita com gaze ou fralda umedecida com água, 7\%, com dedeira e 75,5\% com escova dental. Dos acompanhantes que responderam a questão sobre uso do dentifrício, $55,6 \%$ disseram que era utilizado.

Após exame clínico, constatou-se que, em relação à atividade de cárie, $45,3 \%$ dos pacientes eram cárie ativos e $54,7 \%$ não tinham atividade de cárie (Tabela 3 ).

Durante a análise dos prontuários foi feita a contagem de consultas realizadas ao longo desses anos, totalizando uma soma de 2038, com média de 3,63 consultas por paciente. Foram também calculados todos os procedimentos realizados, sendo a instrução de higiene bucal aliada à orientação de dieta, o mais realizado, com uma soma de 1557 (média de 2,74 por paciente), seguido por 550 aplicações tópicas de flúor (média de 0,97 por paciente) e 352 tratamentos restauradores atraumáticos (ART) (média de 0,62 por paciente). Observou-se também uma quantidade significativa de exodontias (128) e aplicações de cariostático (107).

Após a obtenção de todas as frequências e porcentagens simples, analisou-se a associação da forma de ingresso com a faixa etária $(n=555$ e $\mathrm{p}=0,000)$. Assim, nas faixas etárias de 0 a 1 ano e de 1 a 2 anos, houve uma maior frequência de crianças que ingressaram por livre demanda com porcentagens de 70,8\% e $57,3 \%$, respectivamente, contrastando com uma maior frequência de crianças que foram encaminhadas pelas UBS nas faixas etárias de 2 a 3 anos e mais de 3 anos, com porcentagens de $61,5 \%$ e $71,4 \%$, respectivamente (Gráfico 1). Fez-se também a distribuição percentual da atividade de cárie em cada faixa etária $(n=507$ e $\mathrm{p}=0,000)$, sendo encontrados os seguintes resultados: a maioria dos bebês que tinha de 0 a 1 ano $(96,1 \%)$ e de 1 a 2 anos de idade $(61,1 \%)$ era cárie inativa, porém houve uma frequência maior da doença cárie nas faixas etárias de 2 a 3 anos (72,3\%) e de mais de 3 anos (71,1\%) (Gráfico 2).

\section{Discussão}

A Odontologia para bebês resultou oficialmente de uma tendência mundial de atenção odontológica para criança de tenra idade com o estabelecimento de programas de educação, de medidas preventivas e de controle de cárie, atenção primária e 
tratamentos curativos específicos para essas crianças, de acordo com Silva (2007). O desafio de trabalhar com a promoção da saúde no setor público, especialmente com bebês, tem chamado a atenção de pesquisadores envolvidos com a educação em saúde. Vários autores têm abordado a necessidade de se estabelecerem ações práticas voltadas para a educação em saúde, especialmente no campo da odontologia, em centros de saúde pública, além de estabelecimento do atendimento odontológico para crianças (WALTER, FERELLE, ISSAO, 1996).

A faixa etária mais frequente nos prontuários analisados de 2006 a 2011 da Bebê Clínica da FO/ UFRGS foi de 1 a 2 anos de idade (37,1\%), mantendo o padrão dos resultados do estudo semelhante realizado por Figueiredo et al.(ANO). Em 2008, no mesmo programa, e confirmando uma mudança na frequência da faixa etária quando comparado ao estudo realizado há 14 anos por Figueiredo, Rosito e Michel, na mesma clínica, onde a mais frequente foi de 2 a 3 anos de idade. Estes dados demonstram uma evolução nos padrões de atendimento do programa, o que acabou por aproximá-lo das orientações da American Academy of Pediatric Dentistry (AAPD), que recomenda que a primeira visita ao cirurgião-dentista deve ocorrer entre a irrupção do primeiro dente decíduo e o primeiro ano de vida do bebê. Deste modo, a Bebê Clínica da FO/UFRGS vem se adequando a essa normatização, pois houve um aumento significativo do ingresso de bebês com idade entre $0 \mathrm{e}$ 1 ano: de $10 \%$ em 1998 para 20,4\% de 2006 a 2011.

Um ponto fundamental nos programas de assistência odontológica para bebês é a necessidade da educação e da conscientização dos pais sobre a saúde bucal de seus filhos, em razão da enorme influência que a família exerce na definição dos hábitos dietéticos e de higiene bucal da criança (MOURA, 2001). Castro, Teixeira e Modesto (2002) reforçaram essa temática ao constatarem, em seu estudo de caso, a influência do perfil materno na condição de saúde bucal da criança. Ideia essa que deve ser considerada, já que a mãe neste estudo foi a principal acompanhante nas consultas dos bebês $(87,2 \%)$.

Quanto ao perfil dos bebês de acordo com sua forma de ingresso na Bebê Clínica da FO/UFRGS, observou-se que bebês com idade mais avançada, acima de 2 anos, eram provindos das UBS, e bebês com idade até 2 anos, ingressaram por livre demanda.
A partir disso, consideram-se distintas possibilidades que ajudariam a elucidar este fato como, por exemplo: as UBS encaminhariam apenas aqueles casos mais complexos que acabam por envolver uma faixa etária mais elevada enquanto atendem à demanda do bebê com menos idade e com menos experiência de doença ou, até mesmo, estariam referenciando todos os pacientes da sua área de abrangência, que começariam a ser atendidos na Unidade em uma idade mais avançada. Entretanto, há ausência de trabalhos que mostrem a associação da faixa etária com as diferentes formas de ingresso, pois estudos presentes na literatura analisaram apenas a faixa etária mais frequente atendida, como o realizado por Ferreira et al. (2002) na Bebê Clínica da Universidade Luterana do Brasil (ULBRA), em que mais da metade das crianças que ingressaram no programa tinham entre 2 e 3 anos de idade.

A partir da disseminação da vertente de atenção odontológica na primeira infância no Brasil, a procura aos serviços para atividades preventivas tem ocorrido com uma frequência cada vez maior, e os benefícios deste fato certamente serão observados no público-alvo, a médio e longo prazo (OLIVEIRA et al.,1999).

Tal afirmação reforça os achados deste estudo, pois $50,2 \%$ das crianças atendidas entre os anos de 2006 e 2011 ingressaram no programa por motivos preventivos. Sendo assim, a crescente demanda por cuidados odontológicos preventivos associada à procura por atendimento de bebês com cada vez menos idade é um fator extremamente positivo. Neste sentido, Guimarães, Costa e Oliveira em 2003 afirmaram que crianças que, desde cedo, estejam mais familiarizadas com o ambiente odontológico terão a oportunidade de adquirir hábitos saudáveis e, assim, uma melhor qualidade de vida.

Sabe-se que o aleitamento materno é uma fonte de alimento rica em nutrientes necessários para o desenvolvimento sadio do bebê, promovendo sua saúde física e psíquica. O Ministério da Saúde (BRASIL, 2009) recomenda o aleitamento materno exclusivo por 6 meses, sendo que, depois desse período, a amamentação deve ser complementada com outros alimentos até o bebê completar 2 anos de idade, momento aconselhado para o desmame. Os esforços de diversos organismos nacionais e internacionais favoreceram o aumento desta prática ao 
longo dos últimos 25 anos, e os achados deste estudo evidenciaram os resultados dessa iniciativa, pois foi verificado que grande parte dos bebês atendidos $(70 \%)$ teve amamentação materna exclusiva até os seus 6 meses de idade.

Observou-se, neste estudo, que $65,2 \%$ dos bebês atendidos possuíam uma dieta cariogênica, sendo que muitos deles eram alimentados com formulações. Este dado é preocupante, e o paciente que tem uma dieta rica em açúcares requer orientação odontológica específica e constante acompanhamento, uma vez que, de acordo com Silva et al.(2003), há influência dos carboidratos, em especial os açúcares, no aparecimento de lesões de cárie. Portanto, hábitos alimentares inadequados, como a introdução de substâncias adoçadas por meio da mamadeira, estão relacionados à colonização precoce pelo Streptococcus mutans, principal bactéria cariogênica, na cavidade bucal de bebês (SEOW, 1998; MOHAN, et al.,1998).

Além disso, o uso contínuo de mamadeiras no período noturno associado a padrões inadequados de dieta e de higiene bucal relaciona-se diretamente com a presença de cáries (FRAIZ; WALTER, 2001) uma vez que, segundo Brass (1996), quando o bebê adormece há uma diminuição do fluxo salivar, e o leite, ao estagnar na cavidade bucal da criança, coagula e diminui o seu $\mathrm{pH}$.

Ocorre, então, a formação de uma placa cariogênica que, utilizando a lactose como substrato, propicia a desmineralização do dente. Sessenta e oito por cento dos bebês analisados neste estudo eram amamentados durante a noite, dado este que reforça a necessidade de orientações e motivação dos responsáveis pelo bebê, incentivando uma mudança de comportamento para promover saúde bucal.

A cárie é uma doença multifatorial, infectocontagiosa e, como mencionado anteriormente, quando ocorre na dentição decídua em bebês (ECC), exibe lesões de maneira múltipla e de rápida evolução (WALTER; FERELLE; ISSAO, 1996). Ferreira et al. (2002) observaram que $60,8 \%$ dos pacientes atendidos na Bebê Clínica da ULBRA tinham lesões de cárie, da mesma forma, estudo semelhante a este, realizado por Figueiredo, Rosito e Michel na Bebê Clínica da FO/UFRGS em 1998, mostrou que 61,5\% dos bebês eram considerados cárie ativos. Já no presente estudo, o percentual de pacientes com esta mesma característica diminuiu para 45,3\%, mos- trando uma queda importante na frequência da doença num período de 13 anos.

Observou-se também que houve um crescimento nos índices de cárie com o avanço da idade dos pacientes. A faixa etária com maior frequência de cárie encontrava-se entre 2 e 3 anos $(72,3 \%)$ e a com menor frequência entre 0 e 1 ano (3,9\%). Tal achado é corroborado por estudos realizados por Fraiz e Walter (2001) e de Bonecker (1996), ao constatarem que o aumento da frequência de cárie em bebês está diretamente relacionado com a idade da criança, provavelmente devido a um maior número de superfícies dentárias expostas às alterações de $\mathrm{pH}$ bucal.

A presença de placa visível nos dentes de bebês é considerada um indicador do risco de cárie ao passo que vários estudos demonstraram a correlação existente entre a presença de biofilme e o risco de ECC, (BECK, et al.,1995; ROETERS, et al.,1992).

Nesse sentido, a escovação tem papel importante na prevenção dessa doença, devido à remoção mecânica do biofilme, embora haja pouca evidência para apoiar a noção de que a escovação dos dentes, por si só, reduz a cárie (TINANOFF; KANELLIS; VARGAS, 2002). O presente estudo observou que $88,6 \%$ dos acompanhantes afirmavam realizar higiene bucal dos bebês, informação essa que contrastou com os achados relacionados à presença de lesões de cárie na população estudada $(45,3 \%)$. Sendo assim, supõe-se que a situação relatada pelos acompanhantes não condizia com a realidade do seu dia a dia, ou o conhecimento e/ou a sua capacidade a respeito da técnica correta de higiene bucal era insuficiente.

Ainda no quesito higiene bucal, segundo Burt e Eklund (1999), o uso de dentifrício fluoretado associado à escovação em bebês é comprovadamente eficaz no controle da ECC. A AAPD recomenda que o uso de dentifrício fluoretado deva iniciar assim que os dentes decíduos irromperem, pelo menos 2 vezes ao dia juntamente com uma escova de dentes macia e de tamanho apropriado à cavidade bucal do bebê, com quantidade ideal de dentifrício equivalente a uma "mancha" nas cerdas da escova para crianças menores de 2 anos e o tamanho de uma "ervilha" para crianças maiores de 2 anos. Observouse que 55,6\% dos bebês atendidos na Bebê Clínica entre 2006 e 2011 tinham contato com dentifrício regularmente. 
Walter e Nakama (1992) afirmaram existir uma relação direta entre hábitos alimentares inadequados e higiene bucal deficiente no surgimento de lesões de cárie. Portanto, é fundamental que os responsáveis pelo bebê compreendam essa relação e passem a inserir mudanças favoráveis à saúde bucal da criança desde seu nascimento. $\mathrm{O}$ fato de que, em quase todas as consultas realizadas na Bebê Clínica da FO/UFRGS, os acompanhantes dos bebês receberam orientações de dieta e higiene bucal, sustenta o propósito central preventivo- educativo deste programa, de maneira que todos os cuidados necessários para favorecer a saúde bucal possam ser compreendidos e colocados em prática.

Infelizmente, há uma carência muito grande por trabalhos semelhantes a este na literatura odontológica nacional e internacional que permitam estabelecer comparações entre resultados de estudos realizados por diversos autores em diferentes períodos. Deste modo, é evidente a necessidade de maior produção científica de qualidade na área de odontologia para bebês com foco nas iniciativas de prevenção e promoção de saúde e que aborde também questões clínicas destes pacientes.

Por fim, ressalta-se a importância da existência de uma ação de extensão consolidada há 17 anos dentro de uma instituição de ensino que possibilita aos alunos de graduação da FO/UFRGS uma formação completa no que se refere não apenas à capacitação dos futuros cirurgiões-dentistas para o atendimento a bebês mas também os inserindo como atores numa perspectiva de promoção de saúde.

\section{Conclusão}

A partir do estudo realizado, estabeleceu-se um determinado perfil aos pacientes atendidos na Bebê Clínica entre os anos de 2006 e 2011: os bebês tinham de 1 a 2 anos e de 2 a 3 anos de idade, frequentemente, e vinham acompanhados de suas mães. Quanto à forma de ingresso no programa, as crianças de 0 a 2 anos chegavam principalmente por livre demanda, e aquelas com mais de 2 anos, por encaminhamentos das UBS. O principal motivo da consulta foi prevenção. Em seu histórico médico, a grande maioria dos bebês teve aleitamento materno exclusivo até os 6 meses de idade e não possuía histórico de doença sistêmica, tendo seu estado de saúde classificado como bom. Em relação à dieta, $65,2 \%$ dos bebês possuía dieta cariogênica e quanto a informações sobre saúde bucal, os bebês analisados eram higienizados periodicamente pelos seus pais, principalmente com o uso da escova dental. Aproximadamente $54 \%$ dos pacientes foram diagnosticados cárie inativos, e a análise da atividade de cárie por faixa etária mostrou que as crianças de 0 a 2 anos eram frequentemente cárie inativas e aquelas com idade superior a 2 anos, cárie ativas.

\section{Agradecimentos}

Os autores agradecem toda a colaboração e apoio na execução deste trabalho do cirurgião dentista Jorge Artur Michel, membro da equipe executora da Extensão Universitária: Bebê Clínica da Faculdade de Odontologia da Universidade Federal do Rio Grande do Sul.

\section{REFERÊNCIAS}

AAPD. Chicago: American Academy of Pediatric Dentistry. Disponível em: <http://www.aapd.org/>. Acesso em: 26 novembro 2012.

BECK, J.D.; WEINTRAUB, J.A.; DISNEY, J.A.; GRAVES, R. C.; STAMM, J. W.; KASTE, L. M.; BOHANNAN, H. M. University of North Carolina Caries Risk Assessment Study: comparisons of high risk prediction, any risk prediction, and any risk etiologic models. Community Dent Oral Epidemiol. v.20, n.6 ,313-21, Dec. 1992.

BONECKER, M. J. S. Estudo epidemiológico da prevalência, distribuição e grau de afecção de cárie dentária em crianças de 0 a 36 meses de idade do município de Diadema- São Paulo- Brasil. Dissertação (Mestrado). São Paulo (SP): Universidade de São Paulo, Faculdade de Odontologia; 1996.

BRASIL. Ministério da Saúde do Brasil. Secretaria de Atenção à Saúde. Departamento de Atenção Básica. Coordenação Nacional de Saúde Bucal. Projeto SB. Brasil: condições de saúde bucal da população brasileira 2002 -2003: resultados principais. Brasília (DF), 2004. Disponível em: <http://www.apcd.org.br/anexos/projetos sociais/projeto_sb.pdf > . Acesso em: 26 novembro 2012.

Saúde da criança: nutrição

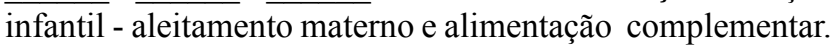
Série A. Normas e Manuais Técnicos Cadernos de Atenção Básica, $\mathrm{n}^{0}$ 23. 2009. Disponível em: $<$ http:// bvsms.saude.gov.br/bvs/publicacoes/saude_crianca nutricao_aleitamento_alime ntacao.pdf $>$. Acesso em: 26 novembro 2012. 
Coordenação Geral de Saúde

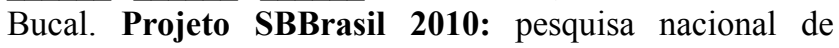
saúde bucal - resultados principais. Brasília (DF), 2011. Disponível em: <http://dab.saude.gov.br/CNSB/sbbrasil/ arquivos/projeto_sb2010_relatorio_final.pdf $>$. Acesso em: 26 novembro 2012.

BRASS, D. Milk: a cause of dental decay. Brit Dent J.,v.20,n.8,p.115-276,1996.

BURT, B. A.; EKLUND, S. A. Dentistry, dental practice, and the community. 5th ed. Philadelphia, Pa: WB Saunders Company; 1999.

CASTRO, L. A.; TEIXEIRA, D. L.; MODESTO, A. A influência do perfil materno na saúde bucal da criança: relato de caso. J Brás Odontopediatr Odontol Bebê, v.5, n.23, p.70- 4, jan/fev, 2002.

ESTRELA, C. Metodologia científica: ensino e pesquisa em Odontologia. São Paulo: Artmed; 2001.

FERREIRA, S. H.; RUSCHEL, H. C.; KRAMER, P. F.; FELDENS, E. G.; SACCOL, K. S. Levantamento dos prontuários da Clínica de Bebês da ULBRA-Canoas RS (1994-2000). Stomatos, Canoas,v. 8, n.15, p.7-14, 2002

FIGUEIREDO, M. C.; GUARIENTI, C. A. D.; MICHEL, J. A.; SAMPAIO, M. S. Comprehensive attention to oral health in early childhood: a longitudinal evaluation of the infant clinic program of the Federal University of Rio Grande do Sul, Brazil. Acta Odontol Latinoam., v.21, n.2, p.181-7, 2008.

FIGUEIREDO, M. C.; ROSITO, D. B.; MICHEL, J. A. Avaliação de 7 anos de um programa odontológico para bebês educativa, preventiva e restauradora. J Bras Odontopediatr Odontol Bebe, v.1, n.2, p. 33-40, abr/ jun, 1998 .

FRACASSO, M. L. C.; MARCHI, V.; GOYA, S.; PROVENZANO, M. G. A.; TAKAHASHI, K. Perfil das mães e crianças freqüentadoras do programa clínica de bebês, no núcleo integrado de saúde NIS III. Iguaçu em Maringá - PR. Revista Saúde e Pesquisa, v.1, n.3, p.325-9, 2008

FRAIZ, F. C.; WALTER, L. R. F. O comportamento infantil durante a higiene bucal domiciliar e alguns fatores associados à cárie. J Bras Odontopediatr Odontol Bebe. v.4, n.21, p.398-404, 2001.

GUimarÃES, A. O.; COSTA, I. C. C.; OLIVEIRA, A. L. $\mathrm{S}$. As origens, objetivos e razões de ser da odontologia para bebês. J Bras Odontopediatr Odontol Bebê. v.6, n.29, p.83-6, 2003

JIN, B. H.; MA, D. S.; MOON, H. S.; PARK, D. I.; HAHN, S. H.; HOROWITZ, A. M. Early childhood caries:prevalence and risk factors in Seoul, Korea. J Public Health Dent., v.63, n.3, p.183-88, 2003.
KOPYCKA-KEDZIERAWSKI, D. T.; AUINGER, P.; BILLINGS, R. J.; WEITZMAN, M. Caries status and overweight in 2- to 18-year-old US children: findings from national surveys. Community Dent Oral Epidemiol., v.36, n.2, p.157-67, 2008.

MOURA, L. F. A. D.; LIRA, D. M. M., MOURA, M. S., BARROS, S. S. L.V.; LOPES, T. S. P.; LEOPOLDINO, V. D.; MOURA, M. D. Apresentação do programa preventivo para gestantes e bebês. J. Bras Odontopediatr Odontol Bebe, Curitiba,v. 4, n.17, p. 10-14,2001.

OLIVEIRA, A. G. R. C.; COSTA, I. C. C.; ARCIERI, R. M.; UNFER, B.; MORAES, E.; SALIBA, N. A. Modelos assistenciais em saúde bucal no Brasil: tendências e perspectivas. Rev. Ação Coletiva, v. 2, n.1, p. 9-14. Jan, 1999.

MOHAN, A.; MORSE, D. E.; O'SULlIVAN, D. M.; TINANOFF, N. The relationship between bottle usage/ content, age, and number of teeth with mutans streptococci colonization in 6-24- month- old children. Community Dent. Oral Epidemiol.,v.26,n.1,p.12-20, Feb,1998.

ROETERS, J.; BURGERSDIJK, R.; TRUIN, G. J.; VAN'T HOF, M. Dental caries and its determinants in 2- to-5-yearold children. ASDC. J Dent Child, v.62, n.6, p. 401-8, Nov/ Dec, 1995.

SAMPAIO, M. S. Tratamento Restaurador Atraumático - ART em bebês afetados pela Cárie Precoce da Infância - ECC: a percepção da mãe. Dissertação (Mestrado). Porto Alegre (RS): Universidade Federal do Rio Grande do Sul, Faculdade de Odontologia, 2005.

SEOW, W. K. Biological mechanisms of early childhood caries. Community Dent Oral Epidemiol., v.26, suppl.1, p. 8-27, 1998.

SILVA, E. L. Odontologia para Bebês. Revista Paraense de Medicina, Belém, v.21, n.4, p.53-6, 2007

SILVA, D. G.; COSTA, B.; GOMIDE, M. R.; TEIXEIRA DAS NEVES, L. T. Breast-feeding and sugar intake in babies with cleft lip and palate. Cleft Palate Craniofac J., v.40, n., p.84-7, jan., 2003.

TINANOFF, N.; KANELLIS, M. J.; VARGAS, C. M. Current understanding of the epidemiology, mechanisms, and prevention of dental caries in preschool children. Pediatr Dent., v.24, n.6, p.543-51, 2002.

WALTER, L. R. F.; NAKAMA, L. Paciente de alto índice de cárie versus paciente de alto risco: qual a conduta? In: BOTTINO, M. A.; FELLER, C. Atualização na clínica odontológica: o dia a dia do clínico geral. São Paulo: Artes Médicas, 1992.

WALTER, L. R. F.; FERELLE, A.; ISSAO, M. Odontologia para o bebê. São Paulo: Artes Médicas, 1996. 